\title{
Difference Between Accessible Social Network and Perceived Social Support in Posttraumatic Growth
}

\author{
Jiaying Xu \\ Department of Psychology, The University of Texas at Austin, Austin, United States
}

Email address:

Jasmine142317@utexas.edu

\section{To cite this article:}

Jiaying Xu. Difference Between Accessible Social Network and Perceived Social Support in Posttraumatic Growth. Psychology and Behavioral Sciences. Vol. 10, No. 3, 2021, pp. 121-124. doi: 10.11648/j.pbs.20211003.13

Received: April 11, 2021; Accepted: June 1, 2021; Published: June 4, 2021

\begin{abstract}
Posttraumatic growth is the positive change in people's thoughts, behaviors, interests among people who were exposed to some serious traumas. Social support has been an important factor for posttraumatic growth, but limited study has looked into the differences between subjectively social support and objectively social support. This paper investigated the relationship between social support (perceived and accessible) and posttraumatic growth. The data were collected from 200 Chinese students through a self-report online survey. The survey includes evaluation of posttraumatic growth, posttraumatic stress disorder, perceived and accessible social support, social network diversity, socioeconomic status, and also trauma history questionnaire. The regression test showed a significant positive correlation between the variables and we further looked into whether there is a difference between perceived social support and accessible social resources. Using the regression test again, we found out that posttraumatic growth is most likely benefit from both perceived SS and objectively accessible social resources. This result suggests that people don't need to have accessible social support as long as they believe they will receive support when they need it, or people do not need to believe they have social support around them as long as the community is providing different types of support. Either of the movements is possible to help people to develop PTG after trauma.
\end{abstract}

Keywords: Posttraumatic Growth, Social Support, Social Network, College Students

\section{Introduction}

Posttraumatic Growth (PTG) is a positive change that might occur after some serious traumatic events [1]. Compared to resilience (i.e., ability to recover when facing trauma), PTG is more like a transformation where people's thoughts, behaviors, interests might all grow or change in a positive way [1]. These positive changes are typically classified into 5 domains: symptoms including appreciation of life, spiritual change, new possibilities, etc. [2]. Researchers have found that PTG is positively associated with ongoing distress, demonstrating that stressful life events could have potentially positive impacts [3]. Moreover, PTG is also a beneficial factor for people's personal life or even psychological disorders like schizophrenia $[4,5]$. Thus, it is important for researchers to investigate the factors that will promote the formation of PTG.

Factors like social support, self-coping style, and work status have all been found positively related to PTG [6]. Among these factors, a handful of studies have found a strong positive association between social support and PTG. For example, Yongju and colleagues [7] found that social support is strongly associated with PTG in a sample of Chinese women with infertility. Another study in Chinese rescue workers showed that social support is partially playing a mediating role between self-acceptance and PTG and it suggested that individuals with a good social support system can help provide more spiritual support for people to cope with trauma [8]. A cross-sectional Cancer study also indicated that social support has a significant positive correlation with PTG which is consistent with the idea that the spiritual support provided by social support can help people better deal with the information from trauma [9]. A study focus on natural hazards also implicates the importance of social support to PTG which they are positively related to each other [10]. However, these studies used an underrepresented sample with restricted types of traumatic events, which limits the generalizability of the conclusion.

Moreover, existing studies have largely overlooked the distinction between subjectively perceived social support and objectively accessible social resources. Theories from social 
cognitive supports the idea that subjectively perceived social support has a greater impact on people's mental health, as perceived support has consequences on how we think about, react to, and subsequently, utilize received support or accessible social resources [11]. In the present study, we operationalize subjectively perceived social support as self-report feelings of supportiveness, and objectively accessible social resources as socioeconomic status and social network diversity, respectively. We will use the Perceived Social Support Scale (PSSS) to measure subjectively perceived social support [12], and objectively accessible social resources will be measured as socioeconomic status and social network diversity. In accordance with the social-cognitive model of social support [11], We hypothesize that compared to objectively accessible social resources, perceived social support will have a stronger association with PTG.

\section{Method}

\subsection{Participants}

Participants were Chinese students from several college chatgroups in Tencent QQ, a popular social media platform in China. Two hundred participants were recruited via online ad and completed the survey. Among them, forty-seven respondents were excluded (three respondents due to low data quality (as assessed with quality control questions) and forty-four due to reporting no trauma history in the Trauma History Questionnaire (THQ). Thus, the final sample size was 153. Due to our mistake in questionnaire formatting, we only collected 34 responses for demographical and socioeconomic status. Respondents received 15 Yuan for their participation (those with low-quality data received only 10 Yuan).

\subsection{Materials}

Perceived social support was assessed with the Chinese version of the PSSS [12]. The scale consists of 12 items measuring subjectively perceived social support. Respondents rated each item on a 7-point Likert scale (1=strongly disagree, $7=$ strongly agree), resulting in a sum score ranging from 12 to 84 (Cronbach's alpha $=0.92)[12]$.

Accessible social resources were defined as participants' social network diversity and socioeconomic status. A more diverse social network and a higher socioeconomic status indicate more accessible social resources.

Social network (SN) diversity was measured with the Chinese version of the Social Network Index Scale (SNI) [13]. The scale consists a total of 12 sets of questions including participants' friends, parents, children, etc. For participants who had connection (at least communicate every two weeks) with the people or groups listed in the question, they received 1 point, so the range of the scores is 0 to 12 points [13].

The socioeconomic status was indexed with participants' and their parents' income, educational level, and occupation. Unfortunately, due to mistakes in survey formatting, most participants did not provide this information, so we had to exclude the socioeconomic status from our analyses.

Trauma history was measured with the Chinese version of the Trauma History Questionnaire (THQ) [14]. We simplified the survey into only asking whether an event had happened before or not where participants received 1 point if they answered yes, resulting in a sum score ranging from 0 to 24 (Cronbach's alpha $=0.60)$ [14].

Posttraumatic growth (PTG) was measured with the Chinese version of the Post Traumatic Growth Inventory (PTGI) [2]. PTGI consists of 21 questions which measure people's change after traumas and the maximum score is 105 . The internal consistency of PTGI in this study is reliable (Cronbach's alpha $=0.95)$. There are 5 different factors in PTGI: Relating to Others (Factor I: item 6, 8, 9, 15, 16, 20, 21; Cronbach's alpha $=0.91)$, new possibilities (Factor II: item 3, 7, 11, 14, 17; Cronbach's alpha =0.87), Personal Strength (Factor III: item 4, 10, 12, 19; Cronbach's alpha $=0.84$ ), Spiritual Change (Factor IV: item 5, 18; Cronbach's alpha = -0.07), and Appreciation of Life (Factor V: item 1, 2, 13; Cronbach's alpha $=0.75$ ). The alpha coefficient in the factor Spiritual Change was negative because there was a non-significant negative correlation between the item 5 ("spiritual life") and the item 18 ("religious faith"). The lack of correlation between the two items may be due to the fact that only a small proportion of Chinese people are religious.

Posttraumatic stress disorder symptoms (PTSD) were measured with the PTSD Checklist for DSM-5 (PCL-5) [15]. The internal consistency of PTSD in this study is reliable (Cronbach's alpha $=0.94)$. This scale consists 20 questions measuring people's posttraumatic stress disorder symptoms. Participants were instructed to rate the frequency of each symptom on a 5 -point Likert scale $(0=$ not at all, $4=$ a lot $)$, resulting in a total score ranging from 0 to 80 . The scale is divided in to four dimensions, namely intrusion (item 1 5; Cronbach's alpha $=0.84$ ), avoidance (item 6 7; Cronbach's alpha $=0.86)$, negative alterations in cognitions and mood (item 8 14; Cronbach's alpha $=0.88$ ), and alterations in arousal and reactivity (item 15 20; Cronbach's alpha $=0.81$; PCL-5).

\subsection{Procedure}

All participants gave electronic signed consent before participation. The survey took approximately 10 minutes to complete. The survey begins with the PTSD checklist, following by perceived social support, THQ, PTGI, accessible social resources, socioeconomic status, and compensation section. Due to our negligence, 162 participants did not provide demographical and socioeconomic information.

\section{Results}

\subsection{Descriptive Statistics}

The means and SDs of all the measures are listed in Table 1 . 
Table 1. Descriptive statistics for predictor and outome variables and correlation among variables.

\begin{tabular}{llllll}
\hline & N & Mean & SD & PTG & SS \\
\hline PTG & 153 & 63.379 & 23.137 & - & - \\
SS & 153 & 57.346 & 12.730 & $0.404 * *$ & - \\
SN_Diversity & 153 & 2.758 & 1.386 & $0.269 * *$ & 0.141 \\
THQ & 153 & 2.797 & 2.263 & 0.007 & - \\
\hline
\end{tabular}

Note. SS = Perceived Social Support Scale; SN Diversity = Social Network Index - diversity; THQ = Trauma History Questionnaire

$* p<0.05$ (two-tailed, uncorrected)

** $p<0.01$ (two-tailed, uncorrected)

\subsection{Confirmatory Analysis}

To test our hypotheses, we used JASP to run the linear regression analysis on 5 predictors (SS, SN diversity, trauma history, interaction between trauma history and perceived social support, interaction between trauma history and social network diversity), and the outcome was PTG.
As show in the table 2, PTG was significantly predicted by $\mathrm{SS}($ Beta $=0.64, b=0.35, t(147)=2.73, p=0.007)$ and social network diversity $($ Beta $=4.32, b=0.26, t(147)=2.22, p=$ $0.028)$. In contrast, THQ and the interactions between THQ and SS and SN diversity were not significant predictors. The overall model was significant $\left(\mathrm{R}^{2}=0.21, p<0.001\right)$.

Table 2. Linear regression results for PTG and predictor variables.

\begin{tabular}{lllll}
\hline Variable & B & SD $(\mathbf{B})$ & B & T \\
\hline Intercept & 13.599 & 13.565 & - & 1.002 \\
SS & 0.639 & 0.234 & 0.352 & 2.731 \\
SN_Diversity & 4.321 & 1.945 & 0.259 & 2.222 \\
THQ & 0.138 & 3.772 & 0.014 & 0.318 \\
Interaction_THQxSS & 0.019 & 0.071 & 0.099 & $0.007 *$ \\
Interaction_THQxSN & -0.254 & 0.468 & -0.110 & 0.265 \\
\hline
\end{tabular}

Note. SS = Perceived Social Support Scale; SN_Diversity = Social Network Index - diversity; THQ = Trauma History Questionnaire.

$* p<0.05$ (two-tailed, uncorrected)

\subsection{Exploratory Analysis}

To test whether perceived social support was a stronger predictor of PTG compared to social network diversity, we conducted a Dunn and Clark's $\mathrm{z}$ test using the "cocorr" package in $\mathrm{R}$ to compare the correlation between social support and PTG and that between social network diversity and PTG. The result indicated that the correlation between social support and PTG $(r=.40)$ was not stronger than that between social network diversity and PTG $\left(r=.27 ; z_{(150)}=1.38\right.$, $p=.1666)$.

\section{Discussion}

PTG is positive changes in people's mental health, thoughts and behaviors after traumatic events [2]. People with more SS tend to have a greater possibility to develop PTG $[8,9]$. However, few studies have looked into the different influences of subjective SS (i.e., self-report feelings of supportiveness) and objective SS (i.e., objectively accessible social resources). This study included measures for both subjective and objective SS and investigated their relationships with PTG. Subjective SS are measured by PSSS and objective SS are measured by SNI $[12,13]$.

Our results showed that both perceived SS and objective social resources were all significantly correlated with PTG. Moreover, in the exploratory analysis, we found that there was no significant difference between the two correlations. This result contradicts our prediction that perceived SS has a stronger relationship with PTG, as has been suggested by the social-cognitive theory of social support [11]. Instead, the result indicates that people perceived few SS could still experience PTG as long as they have a diverse social network. However, the present findings are inconclusive as we failed to measure socioeconomic status, an important facet of objectively accessible social resources. Future research should seek to compare the influence of socioeconomic status and perceived SS on PTG.

\section{Conclusion}

This study investigated the associations between PTG and subjective and objective social support. We found that both perceived SS and social network diversity were correlated with PTG, and there was no significant difference between the strength of the two correlations. Our result suggests that PTG might benefit from both perceived SS and objectively accessible social resources. PTG is important to people's quality of life, especially life after traumas and it is crucial for us to understand what factors can influence PTG. This research can be used in techniques to help people who have been through traumas since even perceived social support is not appearing for people, communities can provide accessible social support which will also promote the growth of PTG. And by providing accessible social support to people, it is possible that people's life quality will increase and the number of people who will be diagnosed with PTSD might decrease. 


\section{References}

[1] Tedeschi, R. G., \& Calhoun, L. G. (2004). Posttraumatic growth: Conceptual foundations and empirical evidence. Psychological Inquiry, 15 (1), 1-18.

[2] Tedeschi, R. G., \& Calhoun, L. G. (1996). The posttraumatic growth inventory: Measuring the positive legacy of trauma. Journal of Traumatic Stress, 9 (3), 455-471.

[3] Loiselle, K. A., Devine, K. A., Reed-Knight, B., et al. (2011). Posttraumatic Growth Associated With a Relative's Serious Illness. Families Systems \& Health, 29 (1), 64-72.

[4] Wu, C., Liu, Y., Ma, S., Jing, G., Zhou, W., Qu, L., Wang, Z., Cheng, M., \& Wu, Y. (2021). The mediating roles of coping styles and resilience in the relationship between perceived social support and posttraumatic growth among primary caregivers of schizophrenic patients: A cross-sectional study. BMC Psychiatry, 21. https://doi-org.ezproxy.lib.utexas.edu/10.1186/s12888-021-03 058-9

[5] Li, Y., Qiao, Y., Luan, X., Li, S., \& Wang, K. (2019). Family resilience and psychological well - being among Chinese breast cancer survivors and their caregivers. European Journal of Cancer Care, 28 (2), 1-8. https://doi-org.ezproxy.lib.utexas.edu/10.1111/ecc.12984

[6] Zhang, L., Lu, Y., Qin, Y., Xue, J., \& Chen, Y. (2020). Post-traumatic growth and related factors among 1221 Chinese cancer survivors. Psycho-Oncology, 29 (2), 413-422. https://doi-org.ezproxy.lib.utexas.edu/10.1002/pon.5279

[7] Yu, Y., Peng, L., Chen, L., et al. (2014). Resilience and social support promote posttraumatic growth of women with infertility: The mediating role of positive coping. Psychiatry Research, 215 (2), 401-405. https://doi-org.ezproxy.lib.utexas.edu/10.1016/j.psychres.2013 .10 .032

[8] Zhao, Y., An, Y., Sun, X., et al. (2020). Self-Acceptance, Post-Traumatic Stress Disorder, Post-Traumatic Growth, and the Role of Social Support in Chinese Rescue Workers. Journal of Loss \& Trauma, 25 (3), 264-277.

[9] Roohi, S., Salehi, J., Mahmoodzadeh, H., et al. (2020). Relationship of Social Support and Coping Strategies with Post-Traumatic Growth and Functional Disability Among Patients with Cancer: Meditating Role of Health Literacy. Iranian Red Crescent Medical Journal, 22 (3), Article e98347, pp. 1-9.

[10] Boullion, G. Q., Pavlacic, J. M., Schulenberg, S. E., Buchanan, E. M., \& Steger, M. F. (2020). Meaning, social support, and resilience as predictors of posttraumatic growth: A study of the Louisiana flooding of August 2016. American Journal of Orthopsychiatry, $\quad 90 \quad$ (5), https://doi-org.ezproxy.lib.utexas.edu/10.1037/ort0000464

[11] Lakey, B., \& Drew, J. B.. A social-cognitive perspective on social support. In Sourcebook of social support and personality New York, NY, US: Plenum Press, 1997, pp. 107-140.

[12] Zimet, G. D., Dahlem, N. W., Zimet, S. G., et al. (1988). The multidimensional scale of perceived social support. Journal of personality assessment, 52 (1), 30-41.

[13] Cohen, S., Doyle, W. J., Skoner, et al. (1997). Social ties and susceptibility to the common cold. Jama-Journal of the American Medical Association, 277 (24), 1940-1944. < Go to ISI>//WOS:A1997XF08700038.

[14] Hooper, L. M., Stockton, P., Krupnick, J. L., et al. (2011). Development, Use, and Psychometric Properties of the Trauma History Questionnaire. Journal of Loss \& Trauma, 16 (3), 258-283.

[15] Blevins, C. A., Weathers, F. W., Davis, M. T., Witte, T. K., \& Domino, J. L. (2015). The Posttraumatic Stress Disorder Checklist for DSM-5 (PCL-5): Development and initial psychometric evaluation. Journal of Traumatic Stress, 28, 489-498. doi: 10.1002/jts.22059. 